WSRC-RP-92-1030

\title{
LATE WASHING EFFICIENCY (U)
}

WSRC-RP- $-92-1030$

DE93 006568

by M. F. Morrissey

Westinghouse Savannah River Company

Savannah River Site

Aiken, South Carolina 29808

Other Authors:

This paper was prepared in connection with work done under Contract No. DE-AC09-89SR18035 with the U. S. Department of Energy. By acceptance of this paper, the publisher and/or recipient acknowledges the U. S.

Government's right to retain a nonexclusive, royalty-free license in and to any copyright covering this paper, along with the right to reproduce and to authorize others to reproduce all or part of the copyrighted paper. 


\section{DISCLAIMER}

This report was prepared as an account of work sponsored by an agency of the United States Government. Neither the United States Government nor any agency thereof, nor any of their employees, makes any warranty, express or implied, or assumes any legal liability or responsibility for the accuracy, completeness, or usefulness of any information, apparatus, product, or process disclosed, or represents that its use would not infringe privately owned rights. Reference herein to any specific commermil produch, process, or service by trade name, trademark, manufacturer, or otherwise does not necessarily constitute or imply its endorsement, recommendation, or favoring by the United States Government or any agency thereof. The views and opinions of authors expressed herein do not necessarily state or reflect those of the United States Government or any agency thereof.

This repor has been reproduced directly from the best available copy.

Available to DOE and DOE contractors from the Office of Scientific and Technical Information, P.O. Box 62, Oak Ridge, TN 37831; prices available from (615) 576-8401, FTS 626-8401.

Available to the public from the National Technical Information Service, U.S. Department of Commerce, 5285 Port Royal Rd, Springfield, VA 22161. 


\section{Westinghouse Savannah River Company}

Savannah River Technology Center

WSRC-RP-92-1030

Keywords: Filters, Cross Flow, Late Washing

Retention: Lifetime

Page 1 of 5

$\infty$. W. L. Tamosaitis, 773-A

E. W. Holtzscheiter, 773-A

M. D. Boersma, 704-T

L. F. Landon, 704-T

J. C. Marek, 704-T

L. M. Nelson, 773-43A

L. O. Dworyanyn, 779-2A

D. J. McCabe, 676-T

M. L. Meyer, 676-1T

D. N. Burns, 676-T

W. J. Vetsch, 704-S

SRL Records

To: D. L. Fish, 773-A

August 31st, 1992

From: M. F. Morrissey, 676-T YM. Y WM,

Late Washing Efficiency (U)

\subsection{Abstract}

Interim Waste Technology has demonstrated the Late Washing concept on the Experimental Laboratory Filter (ELF) at TNX. In two tests, washing reduced the $\left[\mathrm{NO}_{2}^{-}\right]$from $0.08 \mathrm{M}$ to approximately $0.01 \mathrm{M}$ on slurries with 2 year equivalent radiation exposures and $9.5 \mathrm{wt}$. \% solids. For both washes, the $\left[\mathrm{NO}_{2}{ }^{-}\right]$decreased at rates near theoretical for a constant volume stirred vessel, indicating approximately $100 \%$ washing efficiency. Permeate flux was greater than $0.05 \mathrm{gpm} / \mathrm{ft}^{2}$ for both washes at a transmembrane pressure of $50 \mathrm{psi}$ and flow velocity of $9 \mathrm{ft} / \mathrm{sec}$.

\subsection{Introduction}

The In-Tank Precipitation (ITP) Process decontaminates radioactive waste in Tank 48 by precipitating $\mathrm{Cs}^{137}$ with tetraphenylborate and adsorbing $\mathrm{Sr}^{90}$ with sodium titanate. Concentrated potassium tetraphenylborate/sodium titanate slurry is then transferred to Tank 49 for storage. Waste Management will eventually transfer the slurry to the Defense Waste Processing Facility (DWPF) for vitrification, but while waste remains in Tank 49, the $\left[\mathrm{NO}_{2}^{-}\right]$must remain high to inhibit corrosion. Waste with high $\left[\mathrm{NO}_{2}^{-}\right]$complicates DWPF processes. To avoid complications, the $\left[\mathrm{NO}_{2}{ }^{-}\right]$must be reduced to $0.01 \mathrm{M}$.

The Late Washing Process has been prcposed to reduce the $\left[\mathrm{NO}_{2}^{-}\right]$to $\leq 0.01 \mathrm{M}$. In Late Washing, the KTPB slurry will be washed to reduce the $\left[\mathrm{NO}_{2}^{-}\right]$. Fresh wash water dilutes the nitrite and cross flow filtration removes the wash water after it has reached ambient $\left[\mathrm{NO}_{2}^{-}\right]$.

Interim Waste Technology has demonstrated the Late Washing Process with the ELF, a bench scale 
filtration system (See Attachment 1). Flux tests have been conducted with irradiated slurries to evaluate filter performance at various operating conditions. This report examines the washing efficiency by evaluating permeate flux and water usage.

\subsection{Experimental}

\subsection{Equipment}

The ELF contains a single sintered stainless steel filter. Late Washing is being designed to use an ITP type Filter. The ITP filter compares with the ELF filter in the following ways:

1. ELF filter internal diameter $=3 / 8^{\text {" }}$

ITP filter internal diameter $=5 / 8^{n}$

2. ELF filter length $=167 / 8^{n}$

ITP filter Length $=10^{\circ}$

3. The ELF has 1 element/housing

The ITP filter has 144 elements/housing

4. The ELF permeate/backpulse line is in the center of the housing. The ITP permeate/backpulse line is near the filter exhaust.

5. ELF filter surface area $=0.138 \mathrm{ft}^{2}$

ITP filter surface area $=230 \mathrm{ft}^{2}$

6. Both ELF \& ITP filters are $0.5 \mu$ Mott HyPulse filters.

7 The ELF uses a Wilden M2 double diaphragm pump with Blacoh surge suppressor. ITP and probably Late Washing will use low shear centrifugal pumps.

Based on surface area the ELF is a $1 / 1700$ model of the ITP filter.

\subsection{Cleaning}

Performing experiments reproducibly required starting with a clean filter. Recirculating 1.0 wt. \% oxalic acid and 1.0 wt. \% caustic successfully cleaned the filter to original conditions. Testing deionized water flux prior to every experiment verified cleanliness. A graph demonstrating the clean water fluxes for Experiments A - D appears in Attachment 2.

\subsection{Simulant}

DWPT prepared irradiated and non irradiated slurries. The composition is shown in Attachment 3. Non irradiated slurries contained 500 ppm SURFYNOL®, because non irradiated KTPB slurries foam when recirculated in the ELF. Furthermore non irradiated slurries stratify after sufficient agitation. After precipitation, the slurry will appear white, opaque and uniform, but after pumping or agitating the slurry stratifies. KTPB foam forms on top, sodium titanate falls to the bottom and a clear salt soistion remains in the center. Stratified slurny proves more filterable if the pump draws from the clear solution and less filterable if the pump draws from the bottom or top where the solids loading is high. Adding SURFYNOL\& to non irradiated slurries decreases foaming and stratification.

To irradiate slurries, DWPT exposed them to $3 * 10^{8} \mathrm{rad}$ in a ${ }^{60} \mathrm{Co}$ well. This exposure is equivalent io 2 years self irradiation during storage of slurry containing $36 \mathrm{Ci}{ }^{137} \mathrm{Cs} / \mathrm{gallon}$. Slurries with 2 year exposure and no reprecipitation did not require SURFYNOL@.

\subsection{Flux Tests}

The filter's potential for separating spent wash water from irradiated slurry was evaluated by 
filtering non irradiated and irradiated slurries. The data on non irradiated slurries provided a baseline, while the irradiated slurries represented Late Wash feed. Repeating experiments determined reproducibility.

Transmembrane pressure, flow velocity and slurry composition affect filter performance. To evaluate these parameters several experiments were conducted (Experiments A, B, C, D \& E). Experiments $A$ \& $E$ utilized non irradiated slurries and Experiments $B, C$ \& D utilized irradiated slurries. Each experiment's design was a center point with eight points located around the center of an ellipse (Attachment 4). One hour tests were conducted at each point, manually backpulsing for approximately one second every fifteen minutes with $90 \mathrm{psi}$ air. The design sequence appears in Attachment 5. Each experiment began at the center point, $6 \mathrm{fps} \& 30 \mathrm{psi}$, then tested four points on the ellipse followed by a repetition of the center point. The next four tests occurred at the remaining points on the ellipse and the final test occurred at the center point. All experiments used the same test sequence.

The first eleven tests examined clean filter performance. Previous studies with freshly precipitated KTPB slurry have shown that allowing KTPB slurry to remain static for as little as 1 hour decreases filter performance ${ }^{1}$. IWT refers to this phenomenon as "static membrane formation". The potential for static membrane formation in irradiated slurries was evaluated by allowing slurry to remain static in the ELF for two hours and then repeating the eleven tests. A change in flux would indicate static membrane formation. After the second eleven tests, IWT allowed the slurry to remain static for an additional twenty four hours and then ran another eleven tests.

The flux tests were operated in total recycle, so all concentrations remained constant. Pumping the slurry exposes it to shear and backpulsing with air contributes to foaming, each of which decreases permeate flux.

\subsection{Slurry Washing}

For a perfectly mixed constant volume washing the water volume required to reduce $\left[\mathrm{NO}_{2}^{-}\right]$to $0.01 \mathrm{M}$ is given by the following equation:

$$
\begin{aligned}
& V_{\text {wash water }}=V_{\text {aqueous }}{ }^{*} \operatorname{Ln}\left\{\left[\mathrm{NO}_{2}{ }^{-}\right]\right. \text {initial } \\
& V_{\text {aqueous }}=\text { slurry's aqueous volume }
\end{aligned}
$$

$\mathrm{V}_{\text {aqueous }}$ and $\left[\mathrm{NO}_{2}{ }^{-}\right.$]initial were measured and $\mathrm{V}_{\text {wash water }}$ was calculated. Slurry was washed by connecting the permeate line to the spent wash water vessel and then adding wash water to the process vessel at the rate the filter generated spent wash water. The process vessel was agitated during the first washing, but not the second. Maintaining a constant liquid level in the process vessel verified that the spent wash water left at the rate fresh wash water entered.

Before beginning each wash the $\left[\mathrm{NO}_{2}^{-}\right]$was increased to approximately $0.08 \mathrm{M}$. The wash water volumes required to decrease $\left[\mathrm{NO}_{2}{ }^{-}\right]$from $0.08 \mathrm{M}$ to $0.01 \mathrm{M}$ (in $0.01 \mathrm{M}$ increments) were calculated. The permeate was analyzed after adding these volumes to evaluate the washing efficiency.

\subsection{Results and Conclusions}

The Late Washing Demonstration results appear in the Late Washing Demonstration Datasheet and on the Experiment $C$ Graph (Attachments $6 \& 7$ ). The Washing efficiency was close to theoretical. Washing occurred at room temperature, approximately 50 psi transmembrane pressure and 
approximately 9 fps flow velocity. After Experiment $C$ recirculating 1.0 wt. \% oxalic acid and 1 wt. $\%$ caustic were used to clean the filter back to original condition.

The second washing (Experiment $D$ ) did not use an agitator. Turnover in the Process Vessel was greater then 2 vessel volumes/minute and researchers predicted this rapid turnover would provide sufficient agitation. The slurry did not separate into phases during the wash. The second wash was begun by adding nitrite to increase the $\left[\mathrm{NO}_{2}^{-}\right]$to $0.08 \mathrm{M}$. The analytical results showed only 0.06 $M$, this is probably because not all the nitrite had dissolved when the sample was pulled. The Experiment D Graph (Attachment 8 ) demonstrates $\left[\mathrm{NO}_{2}{ }^{-}\right]$was less than theoretical during the first three samples, probably because nitrite was not dissolved at this time. At the end of the wash the $\left[\mathrm{NO}_{2}{ }^{-}\right]$was approximately theoretical. Experiment $\mathrm{D}$ indicates the importance of thorough agitation.

Flux tests were designed to investigate the potential for static membrane formation. The irradiated slurries used in experiments $C$ and $D$ did not display significant static membrane formation during the flux tests.

\subsection{Error Margins}

The ELF uses a Wilden M2 diaphragm pump, which causes pressures and flows to fluctuate. The surge suppressor suppresses, but does not eliminate fluctuation. Fluctuating pressure and flow make reading pressure and flow gauges difficult Readings will have the following error margins:

Measurement
Concentrate Flow
Permeate Flow (High Flow)
Permeate Flow (Low Flow)
Feed Pressure
Concentrate Pressure
Permeate Pressure
Backpulse Duration
Backpulse Frequency

\section{Measurina Device}

Flow Meter 1

Flow Meter 2

Flow Meter 3

Pressure Gauge 2

Pressure Gauge 3

Pressure Gauge 5

Stop watch

Stop watch
Iypical Value

$1.0-10.0 \mathrm{gpm}$

$0.1 \cdot 3.0 \mathrm{gpm}$

$10-250 \mathrm{ml} / \mathrm{min}$

$15-100 \mathrm{psi}$

$15-100 \mathrm{psi}$

0.75 psi

1 - 4 seconds

5 - 20 minutes
Margin of Error

$\pm 0.1 \mathrm{gpm}$

$\pm 0.1 \mathrm{gpm}$

$\pm 5 \mathrm{mVmin}$

\pm 4 psi

$\pm 3 \mathrm{psi}$

\pm 2 psi

\pm 0.5 seconds

\pm 0.5 seconds

\subsection{Attachments}

\subsubsection{Attachment 1 - Experimental Laboratory Filter Sketch}

4.0.2 Attachment 2 - Water Flux Tests A - D Graph

4.0.3 Aitachment 3 - Slurry Formula

\subsubsection{Attachment 4 - Late Washing Flux Test Design}

4.0.5 Attachment 5 - Late Washing Flux Test Design Datasheet

4.0.6 Attachment 6 - Late Washing Demonstration Datasheet

4.0.7 Attachment 7 - Late Washing Demonstration - Experiment C Graph

4.0.8 Attachment 8 - Late Washing Demonstration - Experiment D Graph

\subsection{References}


$\because \quad \therefore \quad \cdot \quad$.

Page 5 of 5

5.0.1 Reference 1 - M. F. Morrissey, ITP Filter Performance, IWT-LWT-91-107, 10/7/92. 


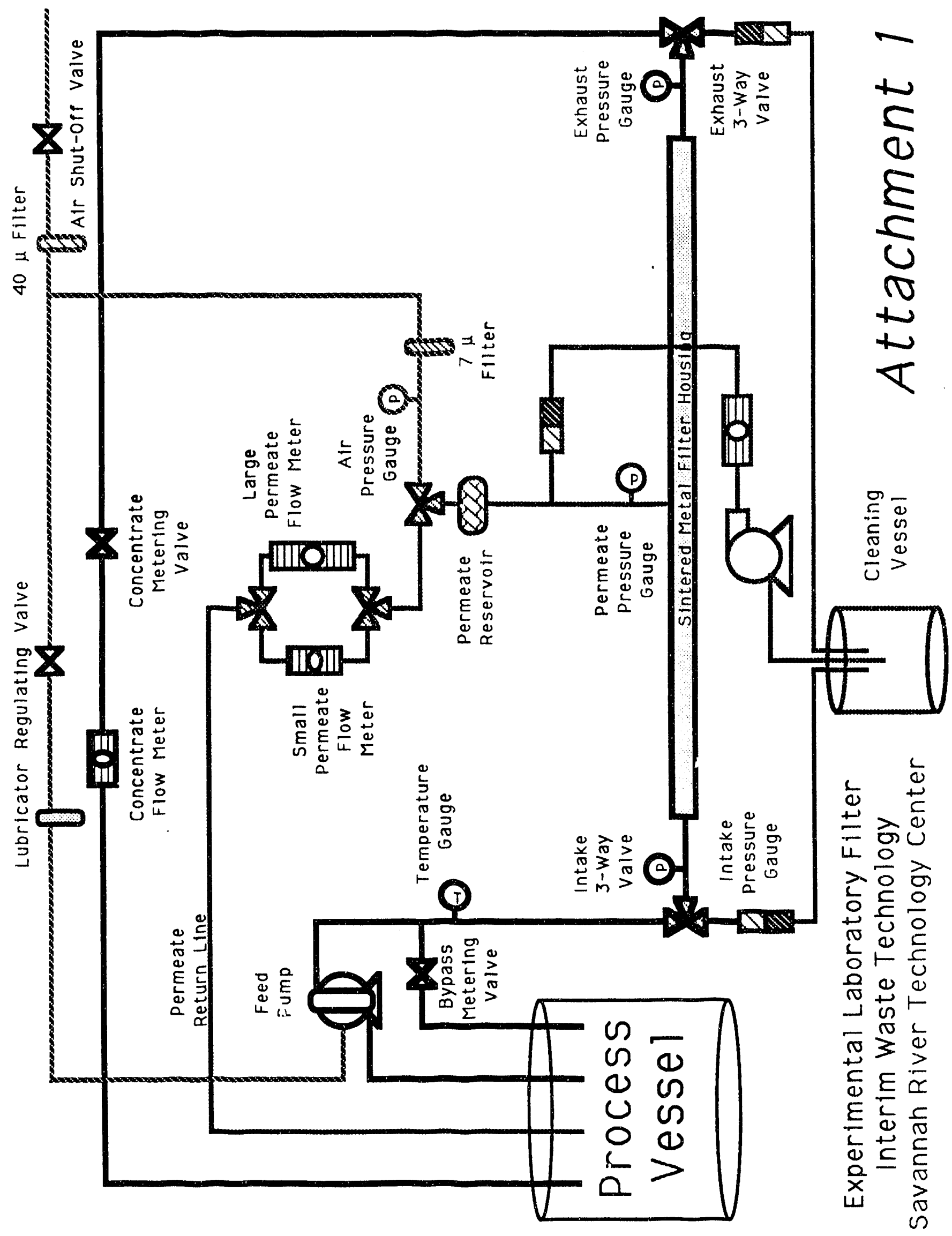




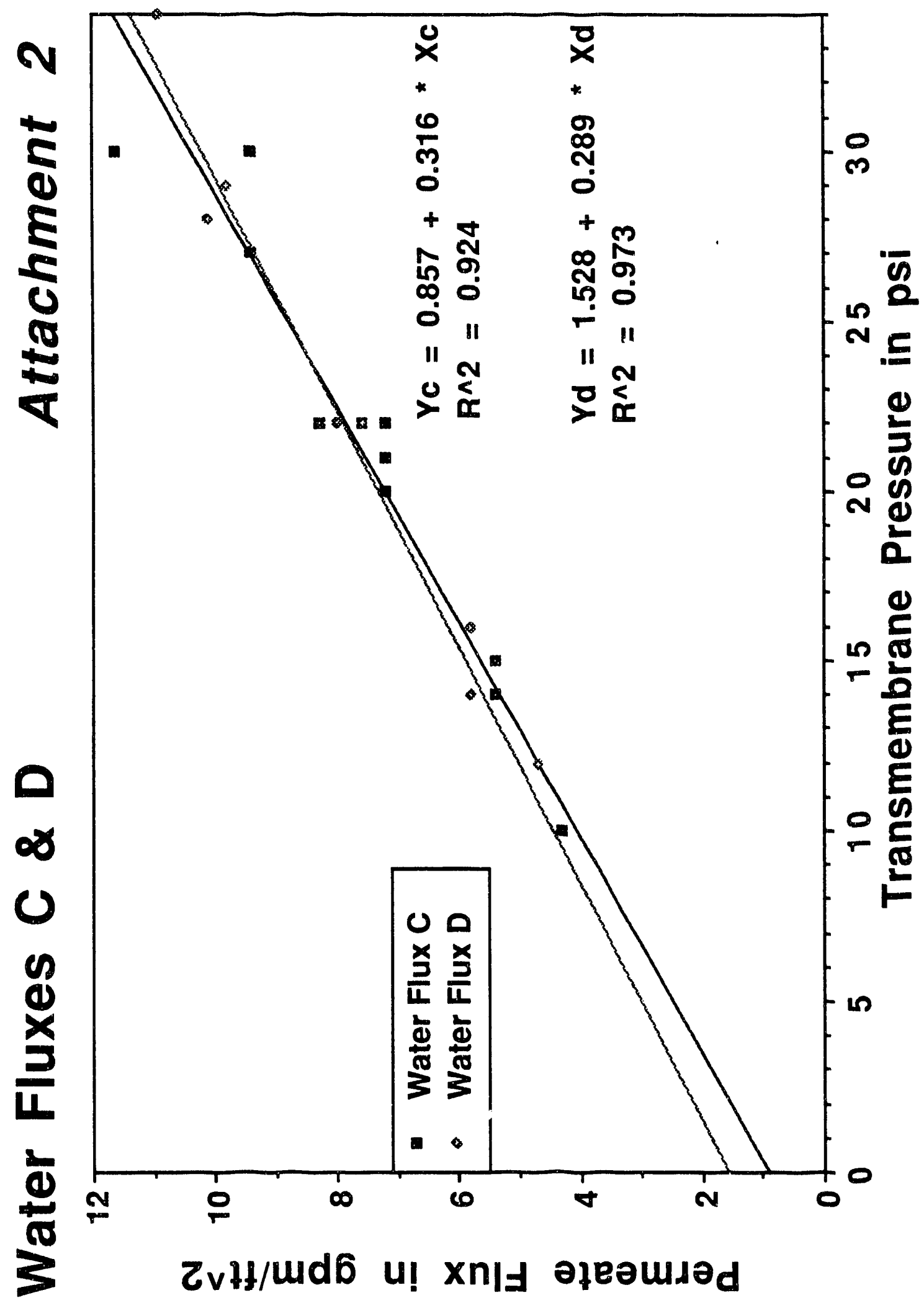


DWPT first blended the salt solution presented below.

\begin{tabular}{|c|c|c|c|c|c|}
\hline Compound & Mass Added & Molecular Welght & Moles & Molarlty & Concentration \\
\hline $\mathrm{H} 2 \mathrm{O}$ & 6.178 .35 grams & $18.00 \mathrm{~g} / \mathrm{mol}$ & 343.2417 moles & $53.9784 \mathrm{M}$ & $952.560 \mathrm{\rho pm}$ \\
\hline $\mathrm{K} 2 \mathrm{CO} 3$ & 3.45 grams & $138.20 \mathrm{~g} / \mathrm{mol}$ & 0.0250 moles & $0.0039 M$ & $532 \mathrm{ppm}$ \\
\hline $\mathrm{KNO2}$ & 268.95 grams & $85.10 \mathrm{~g} / \mathrm{mol}$ & 3.1605 moles & $0.4970 \mathrm{M}$ & $41.466 \mathrm{ppm}$ \\
\hline CsNO3 & 6.72 grams & $194.91 \mathrm{~g} / \mathrm{mol}$ & 0.0345 moles & $0.0054 \mathrm{M}$ & $1.036 \mathrm{ppm}$ \\
\hline $\mathrm{KNO} 3$ & 25.02 grams & $101.10 \mathrm{~g} / \mathrm{mol}$ & 0.2475 moles & $0.0389 \mathrm{M}$ & $3.858 \mathrm{ppm}$ \\
\hline $\mathrm{KCl}$ & 0.24 grams & $74.55 \mathrm{~g} / \mathrm{mol}$ & 0.0032 moles & $0.0005 \mathrm{M}$ & $37 \mathrm{ppm}$ \\
\hline KF & 0.13 grams & $58.10 \mathrm{~g} / \mathrm{mol}$ & 0.0022 moles & $0.0004 M$ & 20 ppm \\
\hline $\mathrm{Na2SO} 4$ & 2.87 grams & $141.98 \mathrm{~g} / \mathrm{mol}$ & 0.0202 moles & $0.0032 \mathrm{M}$ & $442 \mathrm{ppm}$ \\
\hline $\mathrm{K} 2 \mathrm{CrO} 4$ & 0.09 Grams & $194.19 \mathrm{~g} / \mathrm{mol}$ & 0.0005 moles & $0.0001 M$ & $14 \mathrm{ppm}$ \\
\hline $\mathrm{Na2SIO} 3 * 9 \mathrm{H}_{2} \mathrm{O}$ & 0.16 grams & $284.07 \mathrm{~g} / \mathrm{mol}$ & 0.0006 moles & $0.0001 M$ & $25 \mathrm{ppm}$ \\
\hline $\mathrm{K} 2 \mathrm{MnO} 4$ & 0.01 grams & $197.13 \mathrm{~g} / \mathrm{mol}$ & 0.0001 moles & $0.0000 \mathrm{M}$ & $2 \mathrm{ppm}$ \\
\hline $\mathrm{C} 2 \mathrm{H} 5 \mathrm{~B}(\mathrm{OH}) 2$ & 0.06 arams & $94,00 \mathrm{~g} / \mathrm{mol}$ & 0,0006 moles & $0.0001 M$ & $9 \mathrm{ppm}$ \\
\hline
\end{tabular}

\begin{tabular}{l|l|l|l|l} 
Total Mass & 6.486 .05 & grams \\
\hline
\end{tabular}

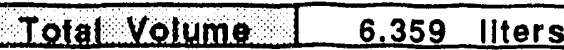

DWPT then added salt solution to NaTPB solution, sodlum titante slurry \& diphenyl mercury.

\begin{tabular}{|c|c|c|c|c|c|}
\hline Slurry & & & & & \\
\hline Compound & Mass 1001 & Molecular Weloht & Moles & Molarity & concentratlon \\
\hline Salt Solution & 3.243 .03 mrams & $N / A$ & $N / A$ & $N / A$ & $N / A$ \\
\hline NaTPB Solution & 3.534 .90 srams & $341.79 \mathrm{~g} / \mathrm{mol}$ & 1.7375 moles & $0.2532 M$ & $504.986 \mathrm{ppm}$ \\
\hline NaT1205H Slurry & 152.50 arsms & $453.73 \mathrm{~g} / \mathrm{mol}$ & 0.0309 moles & $0.0045 M$ & $21.786 \mathrm{ppm}$ \\
\hline$(\mathrm{C} 6 \mathrm{H} 5) 2 \mathrm{Ha}$ & 22.07 grams & $478.18 \mathrm{~g} / \mathrm{mol}$ & 0.0462 moles & $0.0067 \mathrm{M}$ & $3,153 \mathrm{ppm}$ \\
\hline $\mathrm{H} 2 \mathrm{O}$ & 47.50 grams & $18.00 \mathrm{~g} / \mathrm{mol}$ & 2.6389 moles & $0.3845 M$ & $6.786 \mathrm{ppm}$ \\
\hline Total Mass & 7.000 .00 grams & & & Total Volume & 6.863 llters \\
\hline
\end{tabular}

\begin{tabular}{|c|c|c|c|c|c|}
\hline Final Slurry Co & ition & & & & \\
\hline compound & Mass Addod & Molecular weloht & Moles: & Molarity & Concentratlon \\
\hline $\mathrm{H} 2 \mathrm{O}$ & 6.216 .22 grams & $18.00 \mathrm{~g} / \mathrm{mol}$ & 345.3454 moles & $50,3218 M$ & $888,031 \mathrm{ppm}$ \\
\hline $\mathrm{Na}$ & 41.13 grams & $22.99 \mathrm{~g} / \mathrm{mol}$ & 1.7891 moles & $0.2607 \mathrm{M}$ & $5.876 \mathrm{ppm}$ \\
\hline $\mathbf{K}$ & 66.75 grams & $39.10 \mathrm{~g} / \mathrm{mol}$ & 1.7072 moles & $0.2488 \mathrm{M}$ & $9.536 \mathrm{ppm}$ \\
\hline $\mathrm{CO} 3$ & 0.75 grams & $60.00 \mathrm{a} / \mathrm{mol}$ & 0.0125 moles & $0.0018 M$ & $107 \mathrm{ppm}$ \\
\hline NO2 & 47.41 grams & $30.00 \mathrm{~g} / \mathrm{mol}$ & 1.5802 moles & $0.2303 M$ & $6.772 \mathrm{ppm}$ \\
\hline NO3 & 6.48 grams & $46.00 \mathrm{~g} / \mathrm{mol}$ & 0.1410 moles & $0.0205 M$ & $926 \mathrm{ppm}$ \\
\hline $\mathrm{Cl}$ & 0.06 grams & $35.45 \mathrm{~g} / \mathrm{mol}$ & 0.0016 moles & $0.0002 \mathrm{M}$ & 8 ppm \\
\hline $\mathbf{F}$ & 0.02 arams & $20.00 \mathrm{~g} / \mathrm{mol}$ & 0.0011 moles & $0.0002 M$ & $3 \mathrm{ppm}$ \\
\hline SO4 & 0.97 arams & $96,06 \mathrm{~g} / \mathrm{mol}$ & 0.0101 moles & $0.0015 \mathrm{M}$ & $139 \mathrm{ppm}$ \\
\hline CrO4 & 0,03 grams & $116.00 \mathrm{~g} / \mathrm{mol}$ & 0.0002 moles & $0.0000 \mathrm{M}$ & $4 \mathrm{ppm}$ \\
\hline 5103 & 0.02 arams & $76.09 \mathrm{~g} / \mathrm{mol}$ & 0.0003 moles & $0.0000 \mathrm{M}$ & $3 \mathrm{ppm}$ \\
\hline $\mathrm{MnO4}$ & 0.00 grams & $118.94 \mathrm{~g} / \mathrm{mol}$ & 0.0000 moles & $0.0000 \mathrm{M}$ & 0 ppm \\
\hline $\mathrm{C} 2 \mathrm{H} 5 \mathrm{~B}(\mathrm{OH}) 2$ & 0.03 arams & $94.00 \mathrm{~g} / \mathrm{mol}$ & 0.0003 moles & $0.0000 M$ & $4 \mathrm{ppm}$ \\
\hline TPB & 593.86 grams & $341.79 \mathrm{~g} / \mathrm{mol}$ & 1.7375 moles & $0.2532 M$ & $84,838 \mathrm{ppm}$ \\
\hline $\mathrm{T} 12 \mathrm{O} 5 \mathrm{H}$ & 5.46 grams & $176.80 \mathrm{~g} / \mathrm{mol}$ & 0.0028 moles & $0.0004 \mathrm{M}$ & $779 \mathrm{ppm}$ \\
\hline$(\mathrm{C} 6 \mathrm{H} 5)_{2} \mathrm{Hg}$ & 22.07 grams & $478.18 \mathrm{~g} / \mathrm{mol}$ & 0.0462 moles & $0.0067 \mathrm{M}$ & $3,153 \mathrm{ppm}$ \\
\hline
\end{tabular}

Total Mass: $17,000.00$ grams

\begin{tabular}{l|l|l|} 
Total Volume & 6.863 & liters \\
\hline
\end{tabular} 


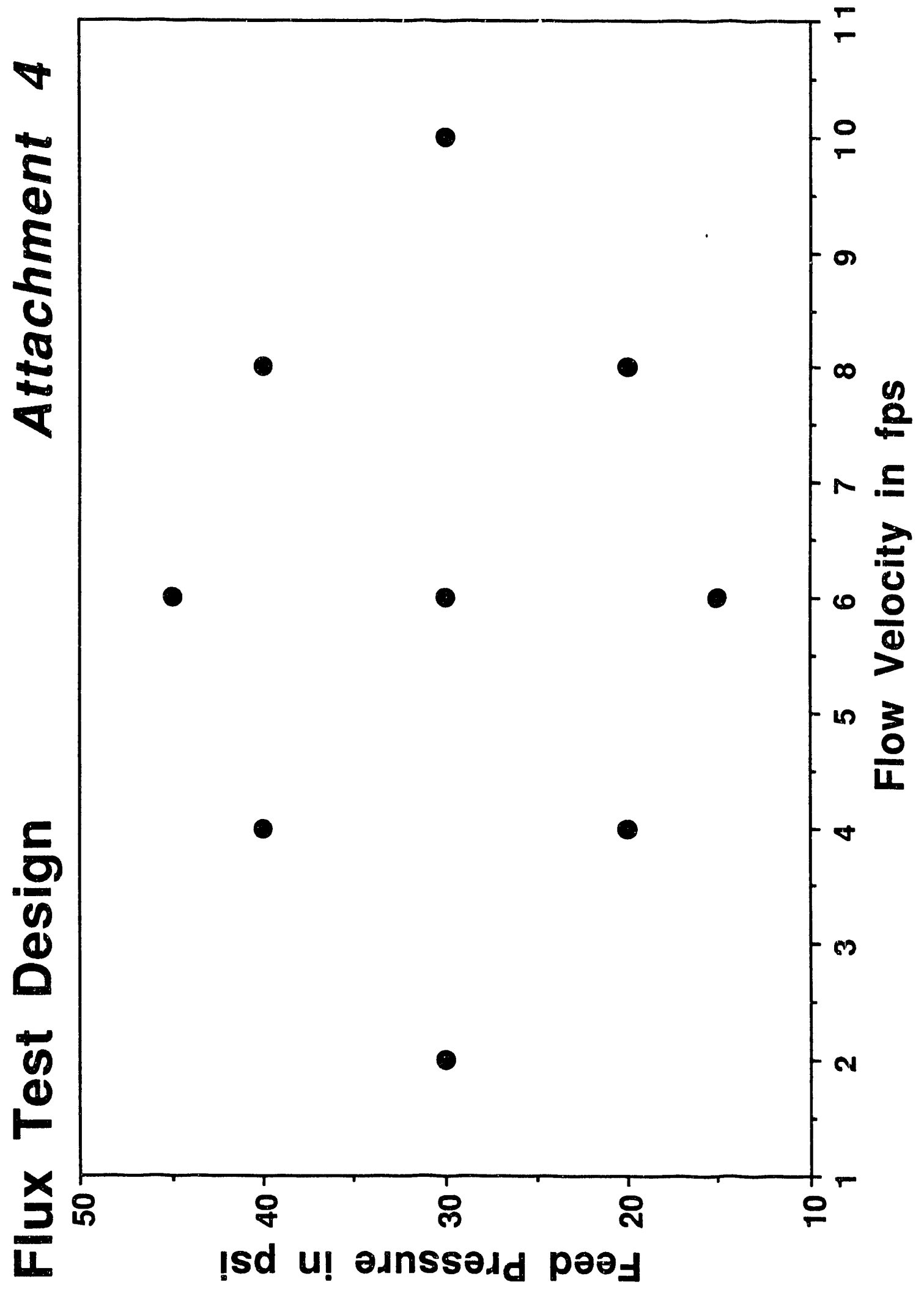




\begin{tabular}{|c|c|c|c|c|c|c|c|c|c|}
\hline \multicolumn{2}{|c|}{ Attachment 5} & & & \multicolumn{5}{|c|}{ Late Washing Flux Test Deslgn Datasheet } & \multirow[b]{2}{*}{$P$ flux } \\
\hline Test & F conc & Vllow & Pleod & $T$ & P conc & F perm & P perm & P trang & \\
\hline 1 & $2.06 \mathrm{gpm}$ & $6 \mathrm{fps}$ & $30 \mathrm{psl}$ & & & & & & \\
\hline 2 & $2.75 \mathrm{gpm}$ & $8 \mathrm{fps}$ & $40 \mathrm{psl}$ & & & & & & \\
\hline 3 & $0.69 \mathrm{gpm}$ & 2 fps & $30 \mathrm{psl}$ & & & & & & \\
\hline 4 & $2.06 \mathrm{gpm}$ & $6 \mathrm{fps}$ & $15 \mathrm{psi}$ & & & & & & \\
\hline 5 & $2.75 \mathrm{gpm}$ & $8 \mathrm{fps}$ & 20 psi & & & & & & \\
\hline 6 & $2.06 \mathrm{gpm}$ & $6 \mathrm{fps}$ & $30 \mathrm{ps}$ & & & & & & \\
\hline 7 & $1.38 \mathrm{gpm}$ & $4 \mathrm{fps}$ & $40 \mathrm{psl}$ & & & & & & \\
\hline 8 & $2.06 \mathrm{gpm}$ & 6 fps & $45 \mathrm{psl}$ & & & & & & \\
\hline 9 & $3.44 \mathrm{gpm}$ & 10 fps & $30 \mathrm{ps}$ & & & & & & \\
\hline 10 & $1.38 \mathrm{gpm}$ & $4 \mathrm{fps}$ & $20 \mathrm{ps}$ & & & & & & \\
\hline 11 & $2.06 \mathrm{gpm}$ & $6 \mathrm{fps}$ & $30 \mathrm{psl}$ & & & & & & \\
\hline \multicolumn{10}{|c|}{2 Hour Statlc Interval } \\
\hline Test & F conc. & Y $110 \mathrm{n}$ & p fead & T. & Pconc. & Pperm & P perm. & Patrans & P flux \\
\hline 12 & $2.06 \mathrm{gpm}$ & $6 \mathrm{fps}$ & $30 \mathrm{psl}$ & & & & & & \\
\hline 13 & $2.75 \mathrm{gpm}$ & $8 \mathrm{fps}$ & $40 \mathrm{psl}$ & & & & & & \\
\hline 14 & $0.69 \mathrm{gpm}$ & 2 fps & $30 \mathrm{psi}$ & & & & & & \\
\hline 15 & $2.06 \mathrm{gpm}$ & $6 \mathrm{fps}$ & $15 \mathrm{psi}$ & & & & & & \\
\hline 16 & $2.75 \mathrm{gpm}$ & $8 \mathrm{fps}$ & $20 \mathrm{ps}$ & & & & & & \\
\hline 17 & $2.06 \mathrm{gpm}$ & 6 ips & $30 \mathrm{ps}$ & & & & & & \\
\hline 18 & $1.38 \mathrm{gpm}$ & 4 fos & $40 \mathrm{p31}$ & & & & & & \\
\hline 19 & $2.06 \mathrm{gpm}$ & 6 fps & $45 \mathrm{psl}$ & & & & & & \\
\hline 20 & $3.44 \mathrm{gpm}$ & $10 \mathrm{fps}$ & $30 \mathrm{psl}$ & & & & & & \\
\hline 21 & $1.38 \mathrm{gpm}$ & 4 fps & $20 \mathrm{psl}$ & & & & & & \\
\hline 22 & $2.06 \mathrm{gpm}$ & $6 \mathrm{fps}$ & $30 \mathrm{psi}$ & & & & & & \\
\hline \multicolumn{10}{|c|}{24 Hour Statlc Interval } \\
\hline Test & F conc. & V flow & P: leed & T & P.conc: & F perm. & P perm & P trans & P flux \\
\hline 23 & $2.06 \mathrm{gpm}$ & $6 \mathrm{fps}$ & $30 \mathrm{psl}$ & & & & & & \\
\hline 24 & $2.75 \mathrm{gpm}$ & $8 \mathrm{fps}$ & $40 \mathrm{psI}$ & & & & & & \\
\hline 25 & $0.69 \mathrm{gpm}$ & $2 \mathrm{fps}$ & $30 \mathrm{psl}$ & & & & & & \\
\hline 26 & $2.06 \mathrm{gpm}$ & 6 fps & $15 \mathrm{psl}$ & & & & & & \\
\hline 27 & $2.75 \mathrm{gpm}$ & 8 fps & $20 \mathrm{ps}$ & & & & & & \\
\hline 28 & $2.06 \mathrm{gpm}$ & $6 \mathrm{fps}$ & $30 \mathrm{psl}$ & & & & & & \\
\hline 29 & $1.38 \mathrm{gpm}$ & 4 fps & $40 \mathrm{psl}$ & & & & & & \\
\hline 30 & $2.06 \mathrm{gpm}$ & $6 \mathrm{fps}$ & $45 \mathrm{ps}$ & & & & & & \\
\hline 31 & $3.44 \mathrm{gpm}$ & 10 fos & $30 \mathrm{psl}$ & & & & & & \\
\hline 32 & $1.38 \mathrm{gpm}$ & 4 tps & $20 \mathrm{psl}$ & & & & & & \\
\hline 33 & $2.06 \mathrm{gpm}$ & 6 fps & $30 \mathrm{psl}$ & & & & & & \\
\hline
\end{tabular}




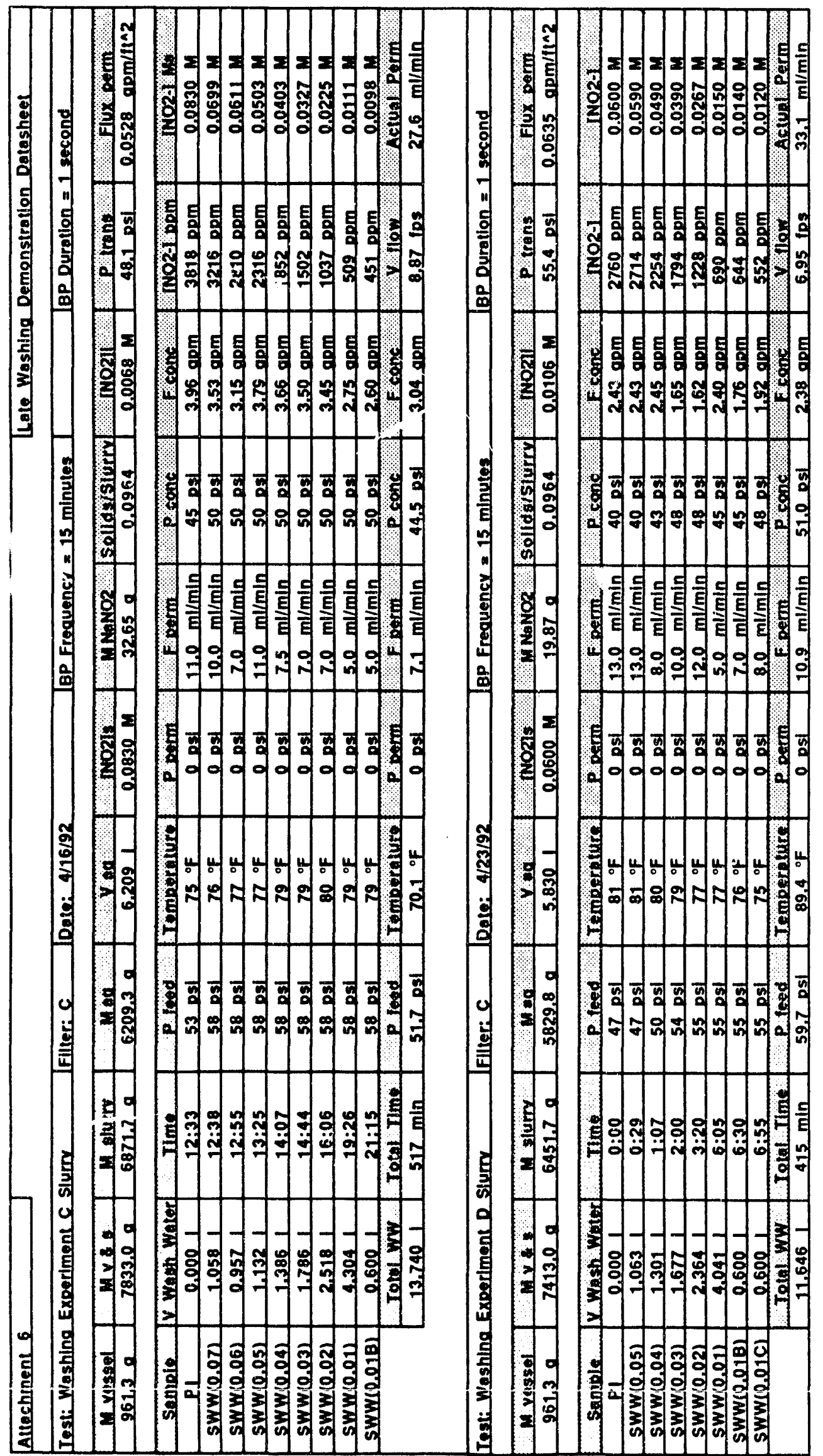




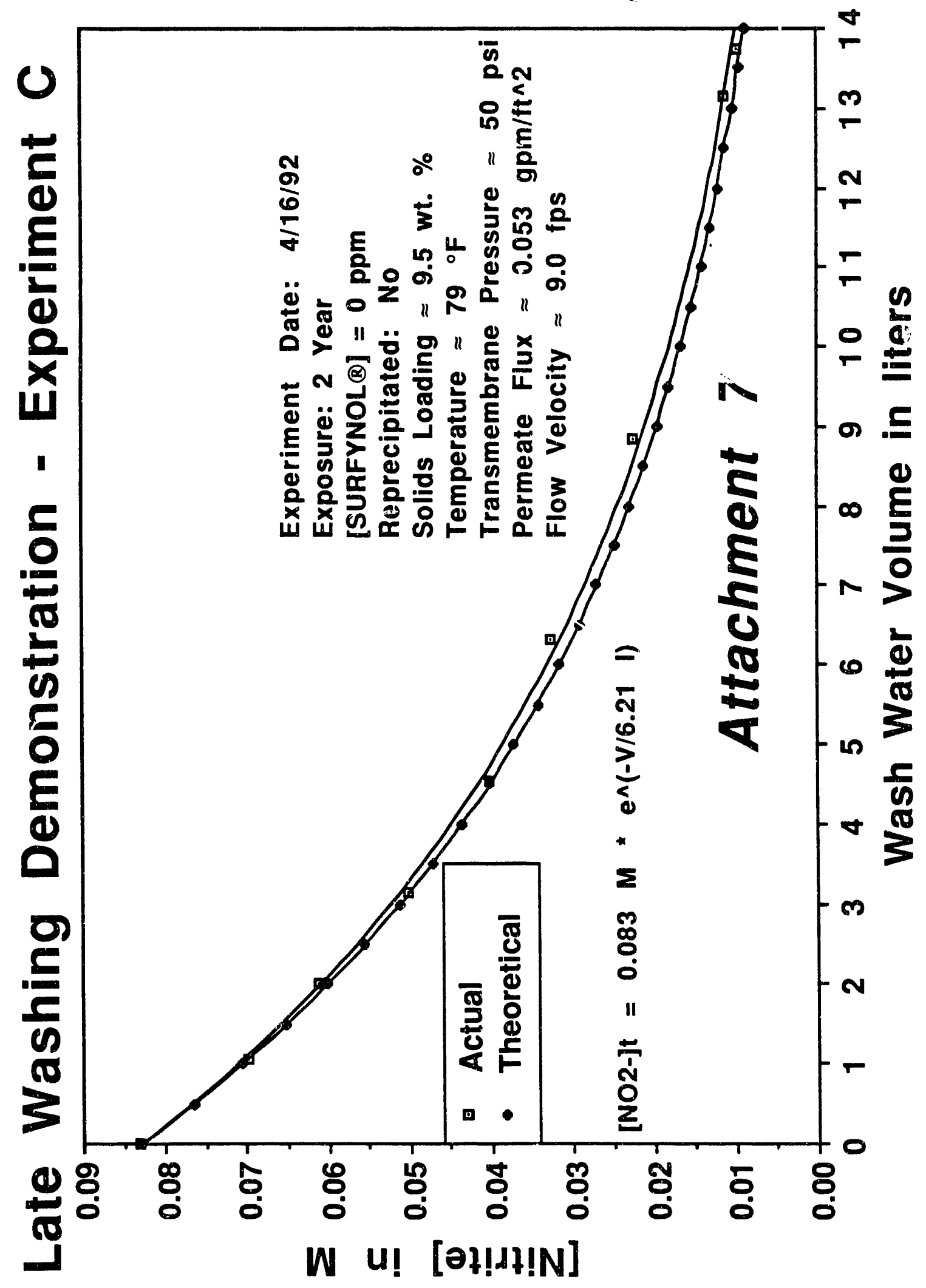




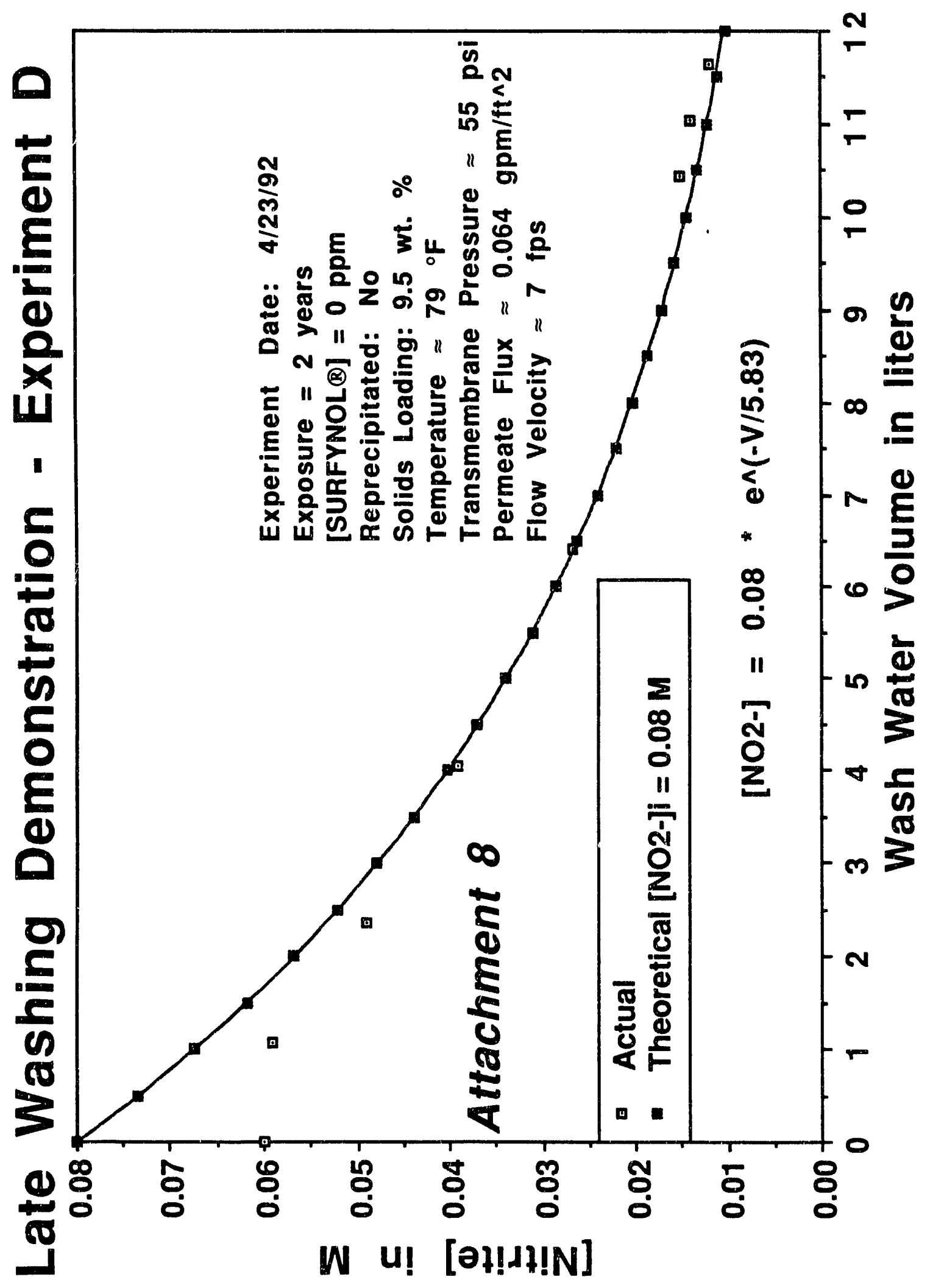



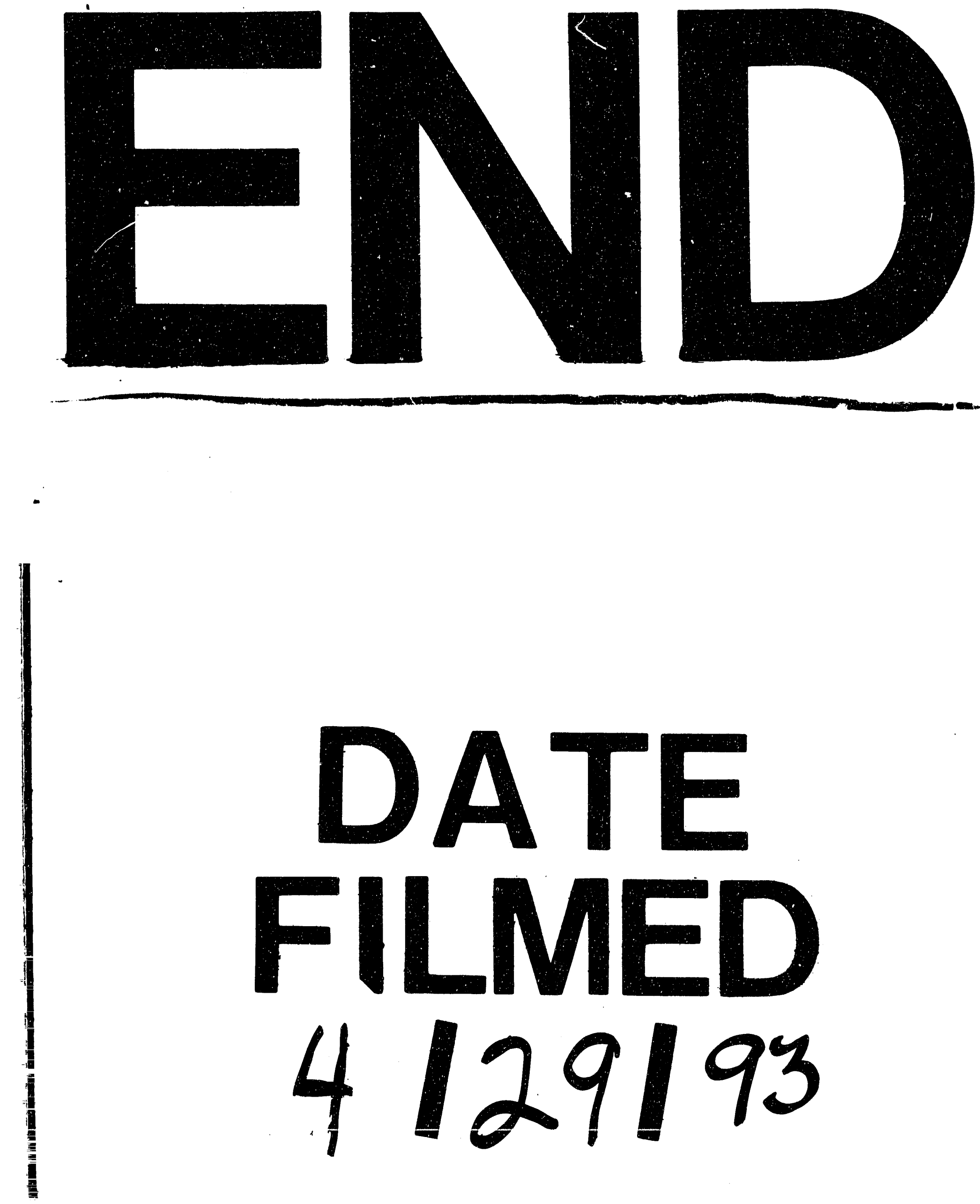
